\title{
Acute Changes in Mitral Valve Geometry after Percutaneous Valve Repair with MitraClip XTR by Three-Dimensional Echocardiography
}

\author{
Maurizio Tusa ${ }^{1}$, Marta Barletta ${ }^{1}$, Antonio Popolo Rubbio ${ }^{1}$, Nicole Travaglio ${ }^{1}$, Silvia \\ Saffioti $^{1}$, Giuseppina Granata ${ }^{1}$, Valentina Mantovani ${ }^{1}$, Elisa Stefanini ${ }^{1}$, Anca Corciu ${ }^{1}$, Luca \\ Testa $^{1}$, and Francesco Bedogni ${ }^{1}$ \\ ${ }^{1}$ IRCCS Policlinico San Donato
}

March 19, 2021

\begin{abstract}
BACKGROUND. Mitral valve (MV) repair with MitraClip system is a safe treatment option for high-risk patients with significant mitral regurgitation (MR). We aimed to characterize, by three-dimensional echocardiography (3D-E), changes occurring in MV after implantation of third generation MitraClip XTR device, with specific reference to the underlying MR mechanism (functional vs. degenerative, FMR vs DMR). METHODS. We prospectively enrolled 59 patients, who underwent intra-procedural 3D-E before and after device deployment. Three-D datasets were analysed off-line, using a dedicated semiautomatic software, to obtain parametric quantification of mitral anatomy. RESULTS. Post-procedural MR of mild or lesser degree was achieved in 40 patients (68\%), with no differences between FMR and DMR (p 0.9). After MitraClip XTR implantation, the FMR group experienced an immediate annular resizing, with reduction of antero-posterior diameter ( $\mathrm{p} 0.024)$ and sphericity index (p 0.017), next to a recovery of physiological saddle-shape, defined by lower non-planar angle ( $\mathrm{p}[?] 0.001)$ and higher annulus height to commissural width ratio (p [?]0.001). On the opposite, the DMR group revealed a significant decrease of maximum annular velocity ( $p$ 0.027), addressing a mechanic effect of the device deployment. Finally, baseline anterior mitral leaflet angle was found as the strongest independent predictor of acute procedural result (OR 0.91, [CI 0.84-0.99], p = 0.030). CONCLUSIONS. MitraClip XTR implantation acts in restoring the original mitral geometry, with distinctive effects according to MR mechanism. Three-D parametric quantification of MV sheds new light on changes occurring in the valvular apparatus, and helps identifying possible new predictors of acute procedural success.
\end{abstract}

Acute Changes in Mitral Valve Geometry after Percutaneous Valve Repair with MitraClip $\mathrm{XT}_{\mathrm{R}}$ by Three-Dimensional Echocardiography

\section{D Mitral Geometry after MitraClip $\mathrm{XT}_{\mathrm{R}}$ deployment}

Maurizio B. Tusa ${ }^{1}$, MD; Marta Barletta ${ }^{1}$, MD; Antonio Popolo Rubbio ${ }^{1}$, MD; Nicole Travaglio ${ }^{2}$, MD; Silvia Saffioti $^{3}$, MD; Giuseppina Granata ${ }^{1}$, MD; Valentina Mantovani ${ }^{1}$, MD; Elisa Stefanini ${ }^{1}$, MD; Anca I. Corciu ${ }^{1}$, MD; Luca Testa ${ }^{1}$, MD, PhD; Francesco Bedogni ${ }^{1}$, MD

1. IRCCS Policlinico San Donato, Department of Cardiology, San Donato Milanese - Milan, Italy

E-mail:tusa.maurizio@libero.it, marta.barletta@outlook.it,_ antoniorubbio@yahoo.it, nicole.travaglio@hotmail.it,_ silviasaffioti@gmail.com,_giusy.granata@gmail.com, valentina.mantovani84@gmail.com, elisa.stefanini@hotmail.it, ancabistrita@yahoo.com, luctes@gmail.com, francesco.bedogni@grupposandonato.it

Address for correspondence: 
Maurizio Barnaba Tusa

Adress: Piazza Edmondo Malan, 2, 20097 San Donato Milanese MI

E-mail: tusa.maurizio@libero.it

Telephone: +393389307149

FAX: 0252774757

\section{Hosted file}

2. ABSTRACT (Echocardiography).pdf available at https://authorea.com/users/402665/articles/ 514457-acute-changes-in-mitral-valve-geometry-after-percutaneous-valve-repair-withmitraclip-xtr-by-three-dimensional-echocardiography

\section{Hosted file}

3. MANUSCRIPT (Echocardiography).pdf available at https://authorea.com/users/402665/articles/ 514457-acute-changes-in-mitral-valve-geometry-after-percutaneous-valve-repair-withmitraclip-xtr-by-three-dimensional-echocardiography

\section{Hosted file}

4. TABLES \& FIGURES (Echocardiography).pdf available at https://authorea.com/users/402665/ articles/514457-acute-changes-in-mitral-valve-geometry-after-percutaneous-valve-repairwith-mitraclip-xtr-by-three-dimensional-echocardiography

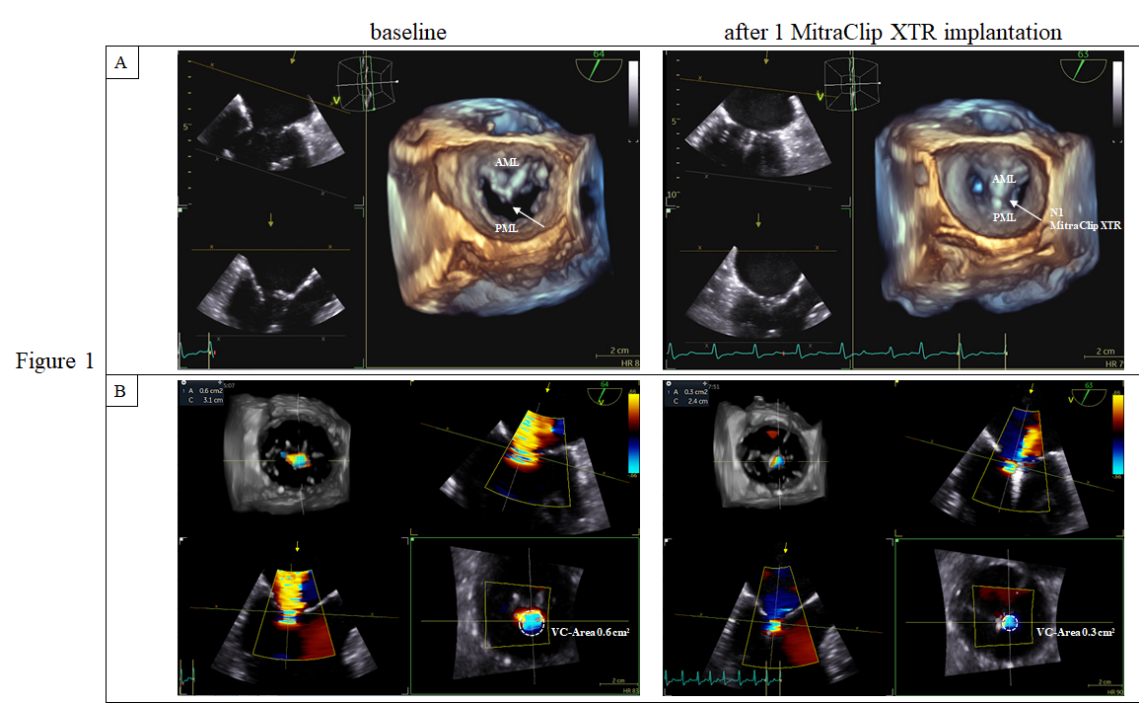



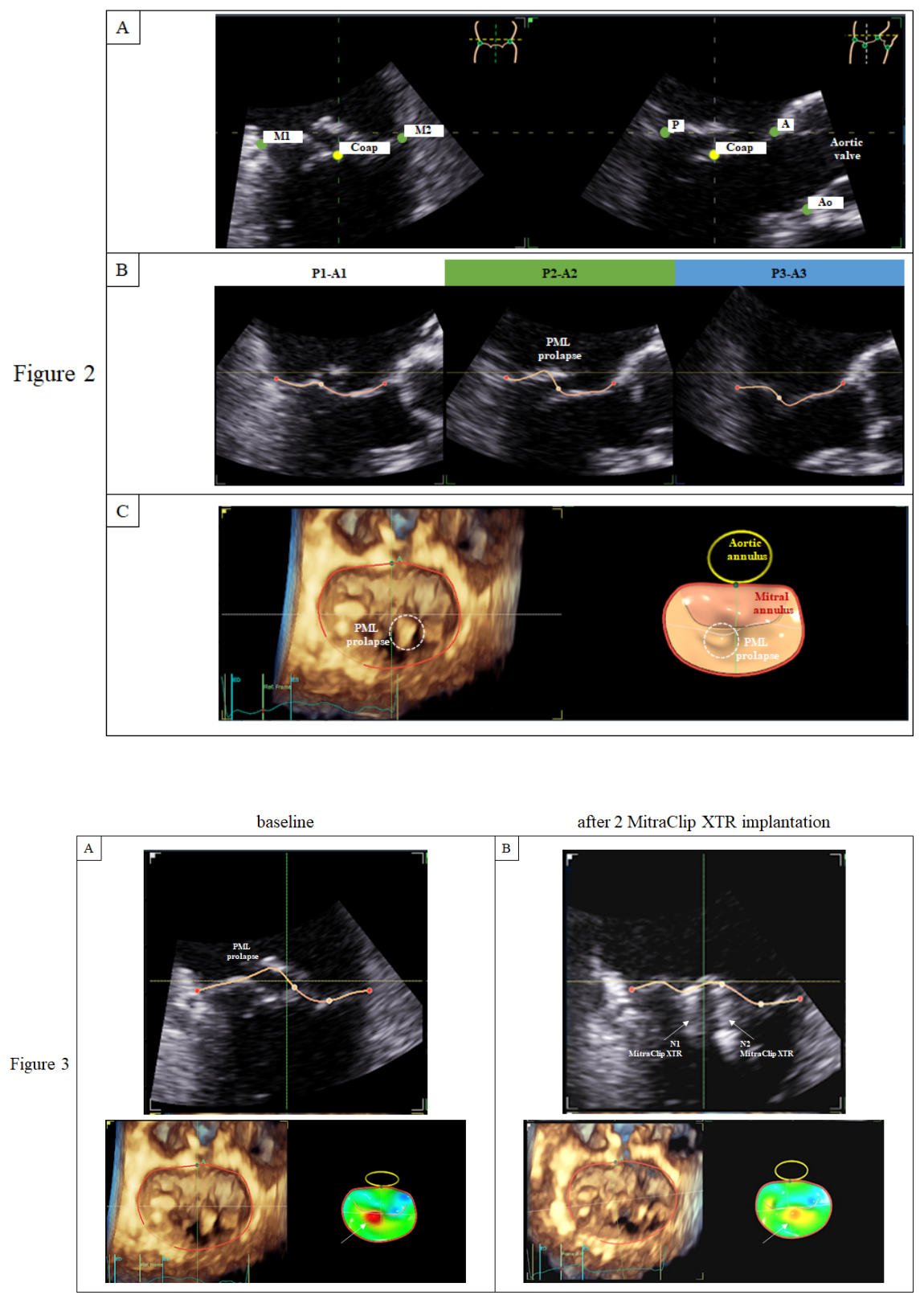